Research Article

\title{
BSA-Embedded Carbonate Apatite Enhances Chemotherapeutic Effect of Paclitaxel in vitro and in vivo
}

Tahereh Fatemian, Ezharul Hoque Chowdhury *

Faculty of Medicine, Nursing and Health Sciences, Monash University, Scenic Blvd \& Wellington Road, Clayton VIC 3800, Australia; E-Mails: tfat1@student.monash.edu, md.ezharul.hoque@monash.edu

* Correspondence: Ezharul Hoque Chowdhury; E-Mail: md.ezharul.hoque@monash.edu

Academic Editor: Tapan K Bera

Special Issue: Molecular Cancer Therapeutics

OBM Genetics

2019 , volume 3 , issue 3

doi:10.21926/obm.genet.1903090
Received: March 29, 2019

Accepted: August 9, 2019

Published: August 15, 2019

\begin{abstract}
Introduction of biocompatible ingredients into nano-material formulations has been studied as a strategy for the enhancement of the pharmacokinetics as well as pharmacodynamics of the final product. With the aim of enhancing drug loading and the ultimate efficacy, incorporation of Bovine Serum Albumin (BSA) into carbonate apatite (CA) nanoparticles structure was employed in the present study. As a result of BSA embedding in the CA formulation, approximately three-times higher loading efficiency was achieved for paclitaxel (Pac). Moreover, BSA-embedded CA complexed with paclitaxel conferred increased efficacy in limiting the viability of cancer cells compared to CA/Pac, and this effect was recorded in breast cancer cell culture as well as in the animal model.

Taken together, with favorable drug binding and suitable in vivo efficacy, BSA-incorporated carbonate apatite may be engineered into a high potency nano-carrier with extensive application in the upcoming research.
\end{abstract}

\section{Keywords}

Carbonate apatite; albumin; drug loading; breast cancer; intravenous delivery

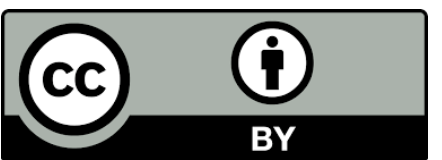

(C) 2019 by the author. This is an open access article distributed under the conditions of the Creative Commons by Attribution License, which permits unrestricted use, distribution, and reproduction in any medium or format, provided the original work is correctly cited. 


\section{Introduction}

Delivery systems have been utilized in the formulations of taxanes in order to confer improvements in the pharmacokinetics and pharmacodynamics of the incorporated therapeutics. Improved biocompatibility and biodegradability, together with increased anticancer efficacy, minimized adverse effects, as well as enhanced water solubility of the taxanes, are among the several advantages of vectorization. Targeted delivery, providing a stable and therapeutically meaningful concentration of the medication at the tumor site along with limited exposure of the normal tissues, is another benefit of the nano-formulation of taxanes. Additionally, nano-vectors may limit the renal clearance of taxanes and enhance their blood accumulation in the host's body, thereby resolving the issue of the short half-life of the taxanes within the body. Nevertheless, in order to ensure the better accumulation of the drug at the tumor site, the ideal taxane delivery system should possess passive or active targeting features to provide further specificity in their distribution to the tumor sites, while minimizing their accumulation in the normal tissues $[1,2]$.

According to the prospective application, nanoparticles may be engineered to contain positively-charged or negatively-charged domains assembled dynamically in each compartment of the vector's structure in order to perform different functions. Various modifications to the constituents of nanoparticles and alterations in the surface characteristics of the vectors may be applied to obtain enhancement in various aspects, including drug loading and biodistribution at the tumor site.

The synthesis process of these delivery systems, as well as the steps for the loading of the drug into these delivery systems, entails a number of details, all of which are required to be meticulously inspected and optimized for the development and establishment of an efficient carrier. Obviously, changes at each step would exert an impact on the nature of the final product, for example on the size, number, and the surface properties of the nanoparticles, which would subsequently impact the dynamics of interaction with the loaded therapeutics and that at the target site.

In this context, several adjustments in the synthesis process of the drug-loaded carbonate apatite nanoparticles were executed and studied in the present study in order to determine the role of each reactant and the synthesis details in the formation of the CA nanoparticles. Additionally, optimization of the prepared formulation of these carriers was performed in the present study.

Complications accompanying the loading of hydrophobic drugs onto nanoparticles include low affinity and rapid release of the drug, in addition to aggregation of the nanoparticles, all of which result in limited internalization of the drug by the cells and increased drug clearance. Numerous attempts have been directed toward altering the surface properties of nanoparticles in order to reduce their clearance by the reticuloendothelial system. Incorporation of various excipients has been attempted for the development of physically stable nanoparticles with their surface characteristics modified through steric and/or ionic stabilization [3-5].

Implemented strategies for achieving improved efficacy, is the surface modification of carbonate apatite with polyethylene glycol (PEG), which results in a drastic reduction in size for the anastrozole-loaded CA. Moreover, the surface modification exhibited an increase in the drug 
uptake, especially for the hydrophobic drug, when compared to conventional CA particles and unbound drug, as confirmed by the HPLC analysis. Similarly, PEGylated carbonate apatite/drug nanoparticle complexes exhibited superior in vitro cytotoxicity and in vivo tumor regression effect when compared to uncoated apatite/drug nanoparticle complexes and unbound drugs [6].

Albumin represents another attractive candidate for the designing of drug delivery systems. Albumin is the most abundant plasma protein and a natural carrier of hydrophobic molecules, which possesses non-covalent binding characteristics, solubility in both water and ethanol, no immunogenicity, and a long half-life. The transport of albumin across the endothelium into the extravascular space is conducted via a cell surface albumin receptor named glycoprotein gp60 and using transcytotic vesicles. Notably, the tumoral tissues actively transport plasma proteins between their cells due to high metabolic demand in these tissues. Currently, three main drug delivery technologies utilizing albumin are available: the conjunction of low-molecular-weight drugs to endogenous or exogenous albumin, the conjugation of albumin with bioactive proteins, and encapsulation of the drug into albumin nanoparticles $[7,8]$. The advantages of this technology include administration of hydrophobic drugs without the requirement of potentially toxic solvents and the selective delivery of greater amounts of the anticancer drug to the tumor sites via endogenous albumin pathways.

Nanoparticle albumin-bound paclitaxel (NAB-paclitaxel), which is the most well-known commercially available medication utilizing albumin, is prepared in a colloidal suspension with an average particle size of $130 \mathrm{~nm}$. It consists of a hydrophobic core that contains the drug which is insoluble, surrounded by a hydrophilic exterior formed by the negatively-charged amino acids present in the unmodified human serum albumin. The novel features of the NAB-paclitaxel formulation have been reported to result in a considerable increase in the intra-tumoral paclitaxel concentrations in an equivalent-dose animal-model study, probably through the exploitation of endogenous albumin pathways $[9,10]$.

In regard to the concerns associated with the in vivo administration of BSA, animal studies on doxorubicin-loaded BSA nanoparticles were conducted, which demonstrated an augmentation in the drug's potency and a decrease in its toxicity, resulting in a significant increase in the average lifetime of the hepatoma tumor-bearing mice [11]. Furthermore, in vivo toxicity studies utilizing intra-peritoneal injections of $\mathrm{BSA}$-coated $\mathrm{Fe}_{3} \mathrm{O}_{4}$ nanoparticles in mice revealed an initial alteration in liver enzymes on Day 7 and 15 post-injection. However, this effect was observed to considerably diminish on Day 30 post-injection, concluding that BSA formulations were relatively safe compared to the other formulations used in the study [12].

In consideration of the aforementioned characteristics of BSA, it was incorporated into the synthesis process of carbonate apatite in the present study, with the aim of promoting drug loading and thereby enhancing the in vitro and in vivo outcomes.

\section{Materials and Methods}

\subsection{Meterials}

Dulbecco's modified eagle medium (DMEM), calcium chloride dihydrate $\left(\mathrm{CaCl}_{2} \cdot 2 \mathrm{H}_{2} \mathrm{O}\right)$, sodium bicarbonate $\left(\mathrm{NaHCO}_{3}\right)$, dimethyl sulfoxide (DMSO), 3-(4,5-dimethylthiazol-2-yl)-2,5-diphenyl tetrazolium bromide (MTT), phosphoric acid solution $\left(\mathrm{H}_{3} \mathrm{PO}_{4}\right)$, trifluoroacetic acid (TFA; $\left.\mathrm{CF}{ }_{3} \mathrm{COOH}\right)$, Ethylene Diamine Tetra Acetic acid (EDTA), and the anti-cancer drug paclitaxel (Pac) were 
purchased from Sigma-Aldrich (St Louis, MO, USA). DMEM powder, fetal bovine serum (FBS), trypsin-ethylene diamine tetraacetate (trypsin-EDTA), 4-(2-hydroxyethyl)-1piperazineethanesulfonic acid (HEPES), and penicillin-streptomycin were obtained from Gibco BRL (California, USA). Acetonitrile (ACN) and triethylamine (TEA) were purchased from Fisher Scientific (Loughborough, UK). All the chemicals used for the HPLC analysis were of HPLC grade. Bovine serum albumin (BSA) was obtained from Bio-Rad. MCF-7, 4T1, and MDA-MB-231 cell lines were originally from ATCC.

\subsection{Cell Culture}

MCF-7 [human breast cancer cell line] and 4T1 [mouse breast cancer cell line] were cultured in DMEM supplemented with $10 \% \mathrm{FBS}, 1 \%$ penicillin-streptomycin, and $1 \% \mathrm{HEPES}$, in $75-\mathrm{cm}^{2}$ tissue culture flasks (Nunc, Orlando, USA) at $37{ }^{\circ} \mathrm{C}$ in a humidified $5 \% \mathrm{CO}_{2}$-containing atmosphere.

\subsection{Synthesis of Carbonate Apatite Nanoparticles}

The synthesis process of CA nanoparticles involved the addition of $\mathrm{Ca}^{2+}$ from $1 \mathrm{M} \mathrm{CaCl}_{2}$ stock solution to the bicarbonate-buffered cell culture medium (DMEM, containing $44 \mathrm{mM} \mathrm{HCO}{ }^{3-}$ and $\mathrm{pH}$ adjusted to 7.5 ) which already contained the third reactant ( $0.9 \mathrm{mM}$ phosphate), followed by incubation at $37{ }^{\circ} \mathrm{C}$ for $30 \mathrm{~min}$. This process resulted in the formation of microscopically visible carbonate apatite (CA) nanoparticles.

\subsection{Characterization of the Synthesized Carbonate Apatite Nanoparticles}

As an indirect approach to investigate the precipitation following the nucleation process in a supersaturated solution, turbidity determination was employed, which interpreted timedependent changes in the optical density in regard to particle formation or growth. In this context, the optical density of the nanoparticle (NP) suspension measured at $320 \mathrm{~nm}$ against a blank (UV1800 UV spectrophotometer, Shimadzu) was associated with the amount of nanoparticles synthesized. The reason for selecting $320 \mathrm{~nm}$ as the wavelength for turbidity measurement of the particle suspension is that at this wavelength, there is the least overlap with the background components of the sample. Size and zeta potential of the variously formulated nanoparticles were measured using the Zetasizer machine (Nano ZS, Malvern) after the addition of $10 \%$ FBS, and the samples were stored at $4{ }^{\circ} \mathrm{C}$ to prevent the formation of aggregates. Zetasizer software 6.20 and a refractive index ratio of 1.325 were utilized to analyze the resulting data.

\subsection{BSA and Drug-Embedded Carbonate Apatite Nanoparticles}

With the aim of enhancing drug interaction efficiency, BSA was introduced into the synthesis process of carbonate apatite as a hydrophilic component which would promote the incorporation of the drug into the structure of nanoparticles. Various amounts of BSA $(0-500 \mu \mathrm{g})$ were incorporated into the NPs formulated with the addition of $7 \mathrm{mM} \mathrm{Ca}$ and $100 \mu \mathrm{M}$ paclitaxel to bicarbonated DMEM, followed by incubation at $37{ }^{\circ} \mathrm{C}$ for $30 \mathrm{~min}$. 


\subsection{Drug Interaction Efficiency}

The drug encapsulation efficiency of the prepared carbonate apatite nanoparticles was analyzed using a High-Performance Liquid Chromatography (HPLC) system equipped with ChemStation software (Agilent Technologies) and an HC-C18 analytical column ( $250 \mathrm{~mm} \times 4.6 \mathrm{~mm}$, $5 \mu \mathrm{m}$; C18, Agilent Technologies). The details of the HPLC method applied for each drug are presented in Table 1.

Table 1 Details of the HPLC method applied for the quantification of each drug.

\begin{tabular}{|c|c|c|c|c|c|}
\hline Drug & Mobile phase & $\begin{array}{c}\text { Flow rate } \\
(\mathrm{ml} / \mathrm{min})\end{array}$ & $\begin{array}{c}\text { Column } \\
\text { temperature } \\
\text { ( } \mathrm{C})\end{array}$ & $\begin{array}{c}\text { Detection } \\
\text { wavelength } \\
(\mathrm{nm})\end{array}$ & $\begin{array}{c}\text { Retention } \\
\text { time } \\
(\mathbf{m i n})\end{array}$ \\
\hline Paclitaxel & $\mathrm{ACN}(+0.1 \% \mathrm{TFA}) / \mathrm{MiliQ}: 70 / 30$ & 1 & 25 & 227 & 2.12 \\
\hline
\end{tabular}

In order to detect the presence of drug within the particles, samples were prepared in bicarbonated DMEM medium ( $\mathrm{pH} 7.5$ ) containing $100 \mu \mathrm{M}$ of each drug with or without $7 \mathrm{mM}$ of exogenous $\mathrm{CaCl}_{2}$. After $30 \mathrm{~min}$ of incubation at $37^{\circ} \mathrm{C}$, samples were centrifuged at $13,800 \mathrm{rpm}$ for $15 \mathrm{~min}$, and the resultant supernatants and pellets were separated. The pellets were re-suspended in $1 \mathrm{~mL}$ DMEM and dissolved with $10 \mathrm{mM}$ EDTA which served as a chelating agent with its sixtoothed structure for binding to metal ions such as $\mathrm{Ca}^{2+}$. After binding by EDTA, the metal ions remained in the solution, although exhibiting diminished reactivity. Three negative controls, which included DMEM only, carbonate apatite only, and the unbound drug in DMEM, were also analyzed using the same method. In order to plot the standard curves, on the basis of which the unknown concentration of the drug in each sample would be calculated, different concentrations $(20,40,60$, 80 , and $100 \mu \mathrm{M}$ ) of each drug were dissolved in the appropriate solvent and analyzed using the exact same method that was used for the determination of drug concentration in the samples. Drug interaction efficiency was calculated using the following formula:

$$
\text { Interaction efficiency }(\%)=\frac{[X]_{C A / d r u g}-[X]_{\text {free drug }}}{[X]_{\text {initial }}} \times 100
$$

where $[X]$ free drug and $[X]_{C A}$ drug represented the amounts of drug present in the pellets (calculated from the standard curves) following the centrifugation of the unbound drug and NPdrug samples, respectively, and $[\mathrm{X}]$ initial represented the amount of drug used initially for the preparation of samples in order to perform HPLC. Each experiment was performed in triplicate, and the results were expressed as mean \pm SD.

\subsection{In vitro Viability Assay}

Cytotoxicity of the prepared carbonate apatite nanoparticles alone and that of the differentlyloaded NPs in human and murine breast cancer cell lines was assessed by performing the MTT assay. 
Briefly, the cells in the exponential growth phase were seeded (approximately 50,000 cells/well) in 24-well plates (Griener, Frickenhausen, Germany), with each well containing DMEM along with $10 \% \mathrm{FBS}$, at $37{ }^{\circ} \mathrm{C}$ and $5 \%-\mathrm{CO}_{2}$ atmosphere. One day after seeding, the cells were treated with the various formulations prepared in the present study, including carbonate apatite samples, BSA/CA, and BSA/Pac/CA. The ingredients were Ca (4 mM), Pac (100 pM, $1 \mathrm{nM}$, and $10 \mathrm{nM})$, and BSA (1 $\mu \mathrm{g}$ and $10 \mu \mathrm{g}$ ). Two days later, cell viability was assessed by the addition of $50 \mu \mathrm{L}$ MTT solution [5 $\mathrm{mg} / \mathrm{mL}$ in phosphate-buffered solution (PBS)] to each well, followed by incubation for $4 \mathrm{~h}$ in dark. Subsequently, the medium was removed, and $300 \mu \mathrm{L}$ DMSO was added to each well to dissolve the purple formazan crystals formed in the previous step. Formazan quantification, in the form of optical density (OD) measurement, was performed at the test and reference wavelengths of 595 $\mathrm{nm}$ and $630 \mathrm{~nm}$, respectively, in a plate reader (Benchmark Plus, Bio-Rad).

Cell viability was calculated using the following formula:

$$
\text { Cell viability }(\%)(C V)=\frac{O D_{\text {(treated) }}-O D_{\text {(reference) }}}{O D_{\text {(untreated) })}-O D_{\text {(reference) }}} \times 100
$$

Reference was the optical density of DMSO only at the applied wavelengths.

Each experiment was performed in triplicate, and the results were expressed as mean $\pm \mathrm{SD}$ of $\%$ cell viability. Subsequently, an increase in the cytotoxic effect of the drugs as a result of complexing with NPs was calculated using the following equation:

Increase in toxicity $(\%)=\mathrm{CV}_{\text {baseline treatment }}-\mathrm{CV}_{\text {complete treatment }}$

where $\mathrm{CV}$ baseline treatment and $\mathrm{CV}$ complete treatment represent the cell viability resulting from baseline treatment and complete treatment, respectively. Increase in toxicity was calculated for all the different concentrations used and was expressed as mean \pm SD.

\subsection{Formulation of Nanoparticles for in vivo Study}

In order to achieve in vivo application, $4 \mu \mathrm{L}$ of $1 \mathrm{mM} \mathrm{CaCl}$ was added to $100 \mu \mathrm{L}$ of freshly prepared bicarbonated DMEM (44 mM) containing $100 \mu \mathrm{g} \mathrm{BSA}$ and $1.25 \mathrm{mg} / \mathrm{kg}$ Pac. The samples were subsequently incubated at $37{ }^{\circ} \mathrm{C}$ for $30 \mathrm{~min}$ and afterward maintained on ice to terminate the further formation of the nanoparticles.

\subsection{T1 Induced Breast Cancer Murine Model}

Female Balb/c mice (6-8 weeks old) weighing 15-20 g were maintained in 12:12 light:dark condition, and were provided ab libitum access to food and water. All the experiments were performed in accordance with the regulations imposed by the Animal Welfare Committee, Monash University. The animal use protocol was approved by the Monash Animal Ethics Committee under protocol number MARP/2012/087.

\subsection{1-Delivery of Anti-Cancer Drugs to Breast Cancer Cells Using Nanoparticles}

Approximately $10^{6}$ of $4 \mathrm{~T} 1$ cells (in $100 \mu \mathrm{L}$ PBS) were injected subcutaneously into the mammary fat pad of mice (considered Day 1), and the mice were examined regularly for the development of tumor by touching the area of injection using index finger. When the volume of the developed 
tumor reached to an average of $13.20 \pm 2.51 \mathrm{~mm}^{3}$, the mice were randomly categorized into different groups, followed by being treated intravenously via right or left caudal vein on Day 11 and 14 . The therapeutics included $100 \mu \mathrm{L}$ of carbonate apatite encompassing $4 \mu \mathrm{L}$ of $1 \mathrm{M} \mathrm{CaCl}_{2}$ and $100 \mu \mathrm{g} \mathrm{BSA}$ with/without $1.25 \mathrm{mg} / \mathrm{kg}$ of Pac (the drug). The mice were closely monitored, and their body weights and tumor outgrowth were recorded each day for a period of 30 days. The tumor volume was calculated using the following formula:

$$
\text { Tumor volume }\left(\mathrm{mm}^{3}\right)=\frac{\left(\text { Length } \times \text { Width }^{2}\right)}{2}
$$

\subsection{Statistics}

In order to determine the statistical significance of the quantifications, Student's t-test was utilized. All the data were expressed as mean \pm SD. Data were considered significant at $p$ values $<0.05$.

\section{Results and Discussion}

\subsection{Incorporation of BSA into CA to Enhance Drug Entrapment}

In this analysis, BSA was incorporated into the synthesis process of the carbonate apatite nanoparticles as a hydrophilic component that would promote the incorporation of the drug into the structure of the nanoparticles (NPs), thereby enhancing the efficiency of the carbonate apatite NPs.

Turbidity measurement was applied as an indirect way to assess the impact of the incorporation of BSA into the CA structure (Figure 1). Complexes were formed using $1 \mu \mathrm{M} \mathrm{Pac}$ together with 5 or $40 \mu \mathrm{g}$ of $\mathrm{BSA}$, followed by incubation at $37{ }^{\circ} \mathrm{C}$ for $30 \mathrm{~min}$. Subsequently, the turbidity of each sample was measured at $320 \mathrm{~nm}$. The sequence of addition of the reactants was also observed. After the addition of paclitaxel, BSA was added in one set of samples prior to $\mathrm{Ca}$, while the other set received the addition of Ca followed by the addition of BSA. The sequence of addition has been represented by the sequence of writing the name of the samples in Figure 1. 


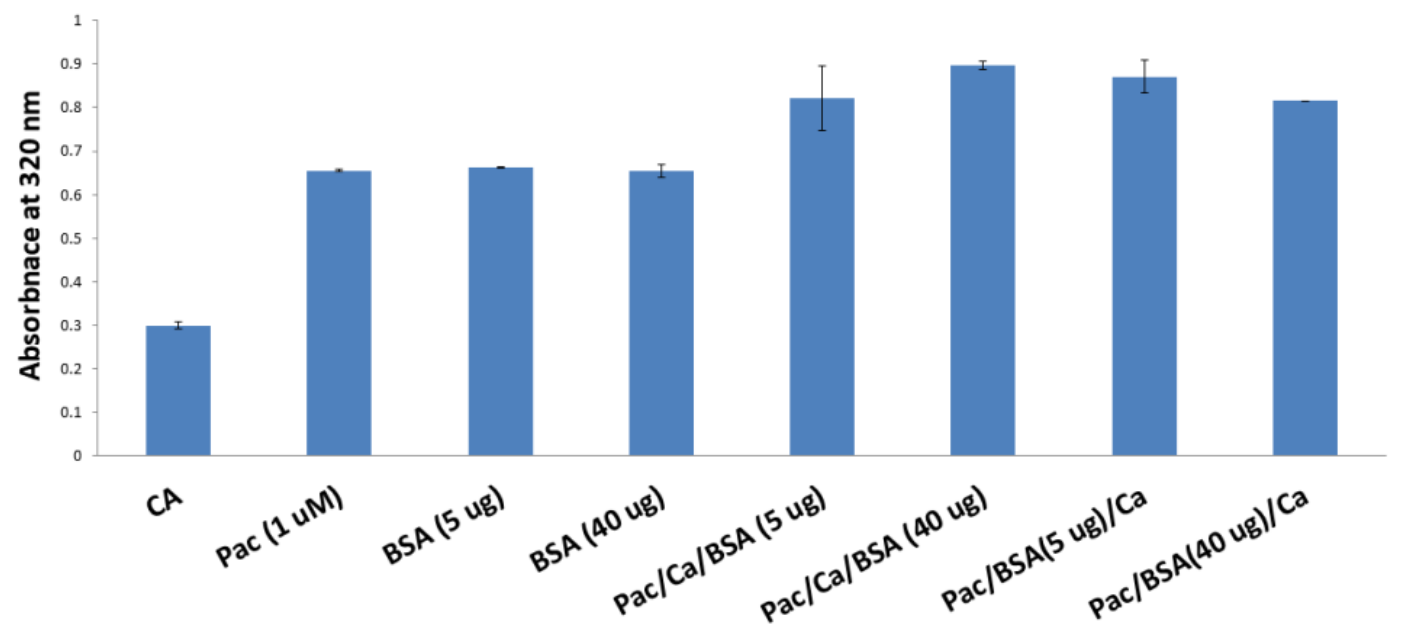

Figure 1 Effect of BSA incorporation into apatite on turbidity. By the addition of $1 \mu \mathrm{M}$ Pac plus $5 \mu \mathrm{g}$ or $40 \mu \mathrm{g} \mathrm{BSA}$, the samples were made and incubated. The absorbance of the samples was then measured at $320 \mathrm{~nm}$ and data are presented as mean \pm SD.

As observed in Figure 1, using $40 \mu \mathrm{g}$ BSA as the last ingredient after Pac and Ca, resulted in highest turbidity, and perhaps, incorporation of greater amounts of drug into the NP complexes.

In order to evaluate the binding efficiency of the BSA-embedded particles prepared in the

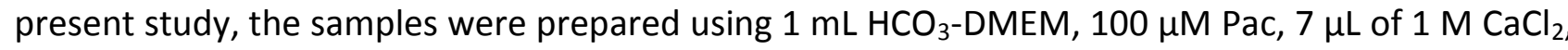
and $0-500 \mu \mathrm{g}$ of BSA. Subsequent to incubation at $37^{\circ} \mathrm{C}$ for $30 \mathrm{~min}$, the samples were centrifuged at $13,200 \mathrm{rpm}$ for $30 \mathrm{~min}$ at $4{ }^{\circ} \mathrm{C}$. After the separation of the resultant supernatants and pellets, 10 mM EDTA was added to all the samples in order to dissolve the structure of apatite.

In terms of the resulting binding efficiency between paclitaxel and BSA-embedded apatite, 50 $\mu \mathrm{g}$ BSA appeared to be the optimal amount of protein, inducing approximately three times increased drug binding (Figure 2). This increase in the drug binding efficiency could be explained by the nature of BSA, which is a highly water-soluble protein, containing charged amino acids that allow the electrostatic adsorption of negatively- or positively-charged molecules. Therefore, it is possible to incorporate substantial amounts of a drug into the CA structure in the presence of BSA. Interestingly, an increase in the amount of the incorporated BSA beyond $50 \mu \mathrm{g}$ did not appear to result in any further enhancement in the drug binding efficiency. The limited and saturable capacity of the binding domains present in the structure of carbonate apatite for the incorporation of BSA could be the reason for this saturation effect. 


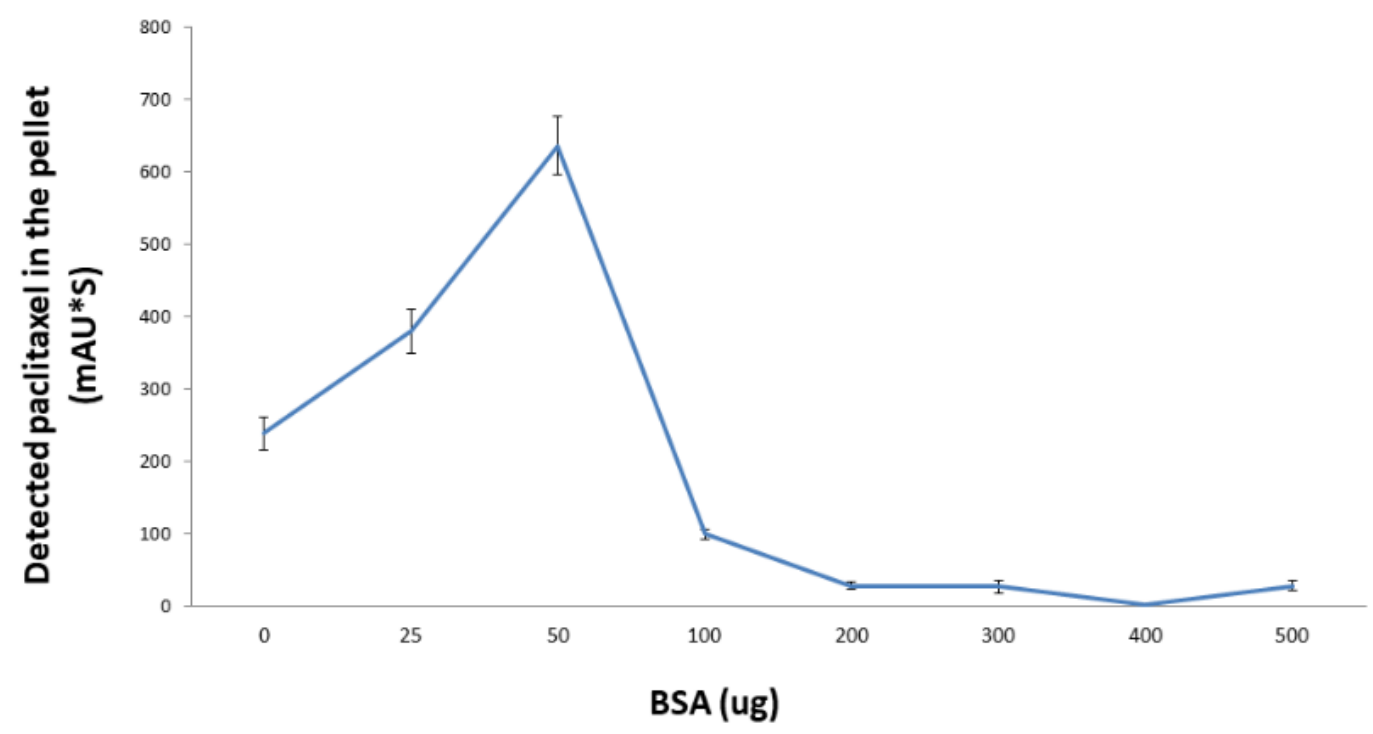

Figure 2 Paclitaxel binding to BSA-embedded apatite particles was measured by incorporating various amounts of BSA $(0-500 \mu \mathrm{g})$ into NPs formulated with the addition of $7 \mathrm{mM} \mathrm{Ca}$ and $100 \mu \mathrm{M}$ of paclitaxel to bicarbonated DMEM followed by 30 min incubation at $37{ }^{\circ} \mathrm{C}$. The resulting NPs were then centrifuged and $10 \mathrm{mM}$ EDTA was added to supernatant and pellet to dissolve the apatite structure. Available paclitaxel in each sample was measured by HPLC. Data are presented as mean $\pm S D, n=3$.

As described in the previous section, the achieved enhancement in the drug encapsulation efficiency following the introduction of BSA into the nano-formulations could be attributed to the electrostatic and hydrophobic interactions between the drug and BSA, followed by the diffusion of the model drug into the BSA matrix $[13,14]$. Nevertheless, the drug is not able to further diffuse into the matrix after the achievement of kinetic equilibrium state. The findings of the present study were consistent with the previously published data available in the literature [13]. Moreover, a sustained release profile has been reported for drug-loaded BSA-containing nanoparticles, indicating an optimal affinity between BSA and the drug together with Fickian diffusion due to electrostatic interaction, which restricted the in vitro release of the positively-charged drug from the negatively-charged BSA [15].

\subsection{Cytotoxic Effects of BSA/Pac/CA}

According to the results of the MTT analysis conducted in MCF-7 cells and the t-test statistics, incorporation of $1 \mu \mathrm{g}$ BSA resulted in a significant enhancement in the cytotoxicity of the $\mathrm{BSA} / \mathrm{Pac} / \mathrm{CA}$ complex at all the concentrations of Pac used in the analysis $(p<0.05)$ (Figure 3$)$. 


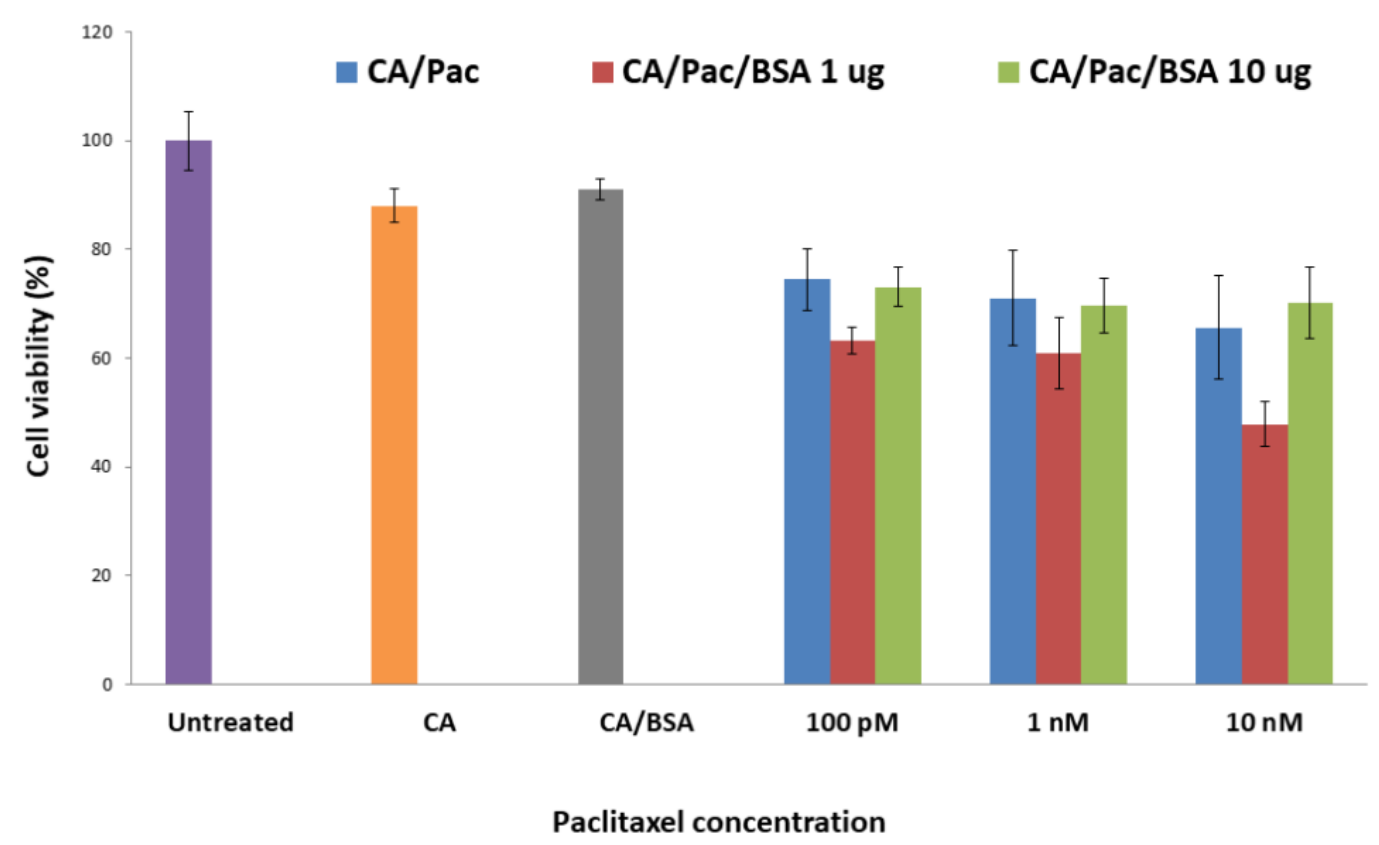

Figure 3 Cell viability assay on MCF-7 cells treated with CA/Pac and CA/Pac/BSA. The cells were seeded on 24 well plates at $5 \times 10^{4}$ cell $/ \mathrm{mL}$. After $24 \mathrm{~h}$ cells were treated with media or free drug or apatite/drug or apatite/drug/BSA. $100 \mathrm{pM}$ to $10 \mathrm{nM}$ of Pac was used for the treatment. Apatite was formed in the presence of the aforementioned drug concentrations using $4 \mathrm{mM} \mathrm{CaCl}_{2}$ and $1 \mu \mathrm{g}$ or $10 \mu \mathrm{g}$ of BSA. After $44 \mathrm{~h}$ of the treatment, cell viability was measured by MTT assay. The values are represented as the percentage of cell viability compared to untreated cells. Data presented as mean \pm SD.

Enhancement in the drug-loading efficiency of the carbonate apatite NPs in the presence of BSA, which was added to improve drug uptake, via endogenous albumin pathways might explain the observed increase in the cytotoxic effects. Again, higher amounts of BSA did not appear to result in any further increase in cytotoxicity, probably due to the limitations in drug binding and uptake in the presence of higher concentrations of BSA. As observed in Table 2, complexing of 10 $\mathrm{nM}$ Pac in addition to $1 \mu \mathrm{g}$ BSA into the apatite structure formed with $4 \mathrm{mM}$ Ca resulted in $17.69 \%$ $\pm 3.53 \%$ increase in cell death in comparison to the complex of CA/Pac.

Table 2 Enhancement in the cytotoxic effect of BSA embedded carbonate apatite carrying Pac into MCF-7 cells compared to apatite bound Pac.

\begin{tabular}{|c|c|c|}
\hline & \multicolumn{2}{|c|}{ Enhancement in cytotoxicity } \\
\hline Pac concentration & $\mathrm{CA} / \mathrm{Pac} / \mathbf{1} \boldsymbol{\mu g}$ BSA & $\mathrm{CA} / \mathrm{Pac} / \mathbf{1 0} \boldsymbol{\mu g}$ BSA \\
\hline $\mathbf{1 0 0} \mathbf{~ p M}$ & $11.25 \pm 3.27$ & $1.45 \pm 0.56$ \\
\hline $\mathbf{1 ~ n M}$ & $10.03 \pm 3.13$ & $1.39 \pm 0.22$ \\
\hline $\mathbf{1 0} \mathrm{nM}$ & $17.69 \pm 3.53$ & $-4.64 \pm 1.04$ \\
\hline
\end{tabular}

The presence of BSA in NPs has been reported to eliminate one of the major drawbacks of the in vitro application of nano-carriers, which is particle aggregation [16], which might serve as an 
explanation for the observed increase in the cytotoxic effects of the BSA-containing carbonate apatite. Additionally, documented sustained release of drug in vitro, for a drug-loaded BSAcontaining nanoparticle suspension [17], may be associated with the increased cytotoxic effects in cell cultures.

\subsection{Effects of BSA Incorporation into Carbonate Apatite Particles and Particle-Bound Paclitaxel on 4T1 Induced-Tumor Mouse Model}

The results of a t-test comparing the anti-tumor efficacy of CA/Pac and CA/BSA/Pac (Figure 4) revealed that the tumor volume in the group receiving BSA-embedded particles was significantly smaller on Day 16 and 18 of the present study. The weight of the animals in all the groups remained approximately unchanged throughout the study.
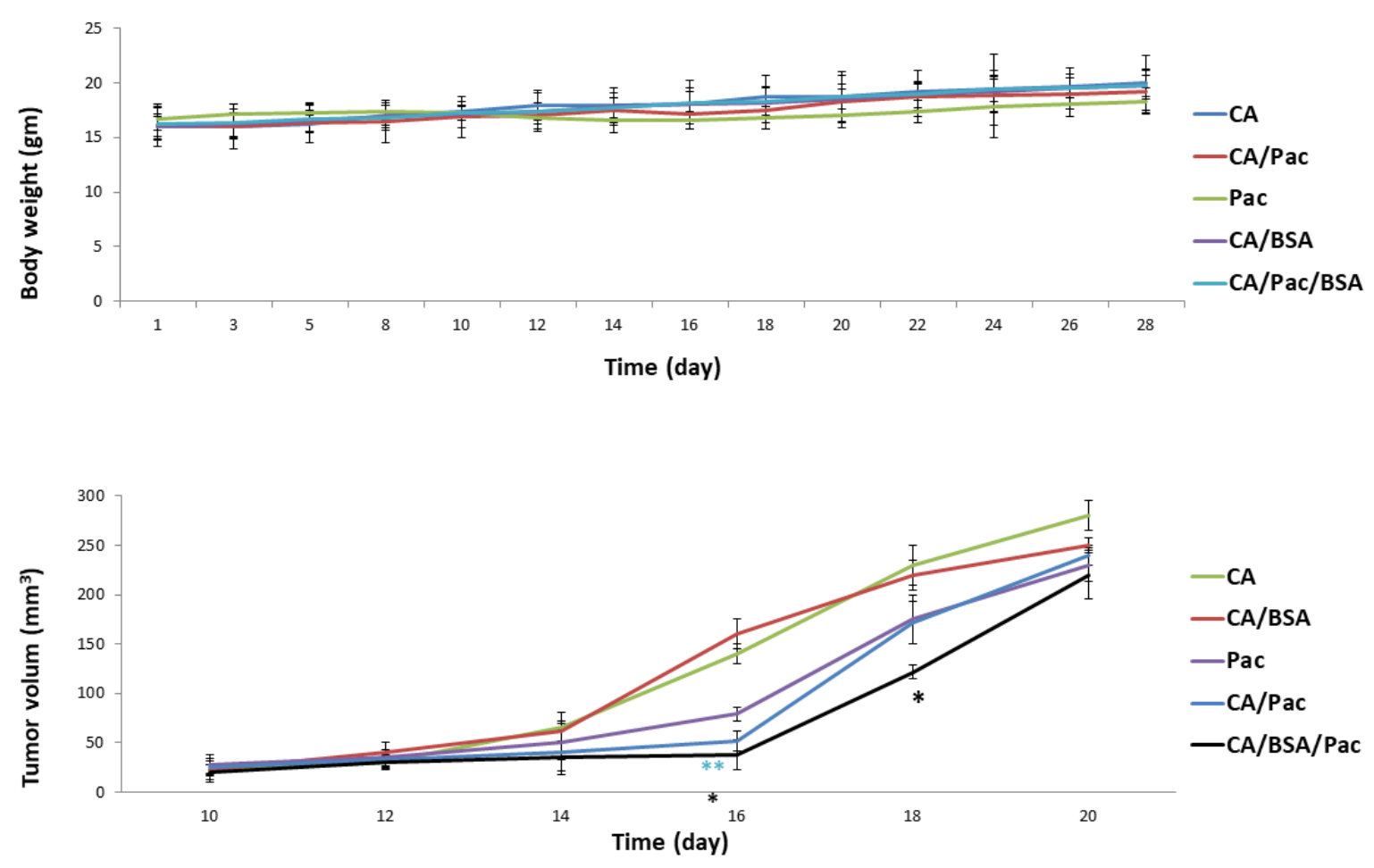

Figure 4 Effect of BSA incorporation into CA/Pac complexes on in vivo tumor regression. The mice were procured from Razi Research Institute, Tehran, Iran. Approximately $10^{6}$ 4T1 cells were inoculated subcutaneously in the mammary pad of mice. Based on tumor volume calculations, mice were randomized and treated intravenously through tail-vein injection on days 11 and 14 . The therapeutics included $100 \mu \mathrm{L}$ of carbonate apatite entailing $4 \mu \mathrm{L}$ of $1 \mathrm{M} \mathrm{CaCl}_{2}$ and $100 \mu \mathrm{g} \mathrm{BSA}$ with/without $1.25 \mathrm{mg} / \mathrm{kg} \mathrm{Pac}$. Bodyweight and tumor outgrowth were monitored every other day. Data are represented as mean $\pm S D, n=6$ and values are significant when * $p$-value $<0.05$ for CA/BSA/Pac compared to CA/Pac group and ** $p$-value $<0.05$ for CA/Pac vs. free Pac.

It is possible to influence the bioavailability of the medications by altering their solubility, as unlike the hydrophilic drugs, hydrophobic drugs benefit from enhanced cellular internalization based on their ability to cross the lipid bilayer of the cell membrane. Conversely, in blood 
circulation, opsonin-mediated endocytosis following the binding of blood plasma protein to hydrophobic molecules results in lower efficacy of the drug [18]. Therefore, albumin might provide a protective hydrophilic domain in the carbonate apatite structure loaded with paclitaxel, thereby increasing the bioavailability of the drug as well as its in vivo efficacy in comparison to the unbound paclitaxel.

Increased drug uptake resulting from enhanced loading of carbonate apatite plus reinforcement via albumin receptors could account for the superior in vivo efficacy of CA/BSA/Pac compared to CA/Pac. In the present study, BSA was introduced into the formulation of drugloaded CA to achieve improved capacity.

According to the published data, increased concentrations of BSA-FITC in plasma were achieved for up to two weeks following the injection of BSA-FITC-loaded microcapsules in rats. Fluorescence images of the microcapsules obtained at different time points revealed a gradual decrease of BSAFITC in the BSA-FITC-loaded microcapsules, confirming a sustained in vivo release of BSA-FITC [19]. Furthermore, in vivo assays conducted on the tumor tissues of mice concluded optimal biocompatibility and efficacy of a BSA template for CuS nanoparticles [20].

In conclusion, incorporation of BSA into apatite structure results in the enhancement of the in vitro and in vivo potentials of drug-loaded carbonate apatite. Augmented loading efficiency added to the fortification of drug uptake at the cellular level via endogenous albumin receptors could account for the observed improvement in efficacy. The increased outcomes documented in the present study might translate into the clinical application of lower doses of taxanes with low side effects in the future. At the same time, higher tumor regression might be achieved using chemotherapy agents at lower doses following the application of novel formulations of CA.

\section{Author Contributions}

These authors contributed equally to this work.

\section{Competing Interests}

The authors have declared that no competing interests exist.

\section{References}

1. Feng L, Mumper RJ. A critical review of lipid-based nanoparticles for taxane delivery. Cancer Lett. 2013; 334: 157-175.

2. Haley B, Frenkel E, editors. Nanoparticles for drug delivery in cancer treatment. UROL ONCOLSEMIN ORI; 2008: Elsevier.

3. Petrak K. Design and properties of particulate carriers for intravascular administration. Marcel Dekker. New York, NY, USA: Rolland, A., 1993.

4. Porter C.J., et al. Microparticulate systems for site-specific therapy bone marrow targeting. In Site-specific Pharmacotherapy. John Willey and Sons. New York, NY, USA: Domb, A., 1994.

5. Elzoghby AO, Samy WM, Elgindy NA. Albumin-based nanoparticles as potential controlled release drug delivery systems. J Control Release. 2012; 157: 168-182. 
6. Mozar F, Chowdhury E. Surface-modification of carbonate apatite nanoparticles enhances delivery and cytotoxicity of gemcitabine and anastrozole in breast cancer cells. Pharmaceutics. 2017; 9: 21.

7. Panyam J, Labhasetwar V. Biodegradable nanoparticles for drug and gene delivery to cells and tissue. Adv Drug Deliv Rev. 2003; 55: 329-347.

8. Guarneri V, Dieci MV, Conte P. Enhancing intracellular taxane delivery: current role and perspectives of nanoparticle albumin-bound paclitaxel in the treatment of advanced breast cancer. Expert Opin Pharmacother. 2012; 13: 395-406.

9. Miele E, Spinelli GP, Miele E, Tomao F, Tomao S. Albumin-bound formulation of paclitaxel (Abraxane ${ }^{\circledR} \mathrm{ABI}-007$ ) in the treatment of breast cancer. Int J Nanomedicine. 2009; 4: 99.

10. Cortes J, Saura C. Nanoparticle albumin-bound (nab $\left.{ }^{T M}\right)$-paclitaxel: improving efficacy and tolerability by targeted drug delivery in metastatic breast cancer. EJC Suppl. 2010; 8: 1-10.

11. Hao $H, M a Q$, Huang $C, H e F$, Yao P. Preparation, characterization, and in vivo evaluation of doxorubicin loaded BSA nanoparticles with folic acid modified dextran surface. Int J Pharm. 2013; 444: 77-84.

12. Hajshafii $P$, Fatahian $S$, Shahanipoor K. In vivo toxicity assessment of bovine serum albumin and dimercaptosuccinic acid coated Fe304 nanoparticles. Iran J Biotechnol. 2014; 12: 63-68.

13. Shi P, Goh JC. Release and cellular acceptance of multiple drugs loaded silk fibroin particles. Int J Pharm. 2011; 420: 282-289.

14. Lammel AS, Hu X, Park S-H, Kaplan DL, Scheibel TR. Controlling silk fibroin particle features for drug delivery. Biomaterials. 2010; 31: 4583-4591.

15. Yu Z, Yu M, Zhang Z, Hong G, Xiong Q. Bovine serum albumin nanoparticles as controlled release carrier for local drug delivery to the inner ear. Nanoscale Res Lett. 2014; 9: 343.

16. Leopold LF, Tódor IS, Diaconeasa Z, Rugină D, Ştefancu A, Leopold N, et al. Assessment of PEG and BSA-PEG gold nanoparticles cellular interaction. Colloid Surface A. 2017; 532: 70-76.

17. Maghsoudi A, Shojaosadati SA, Farahani EV. 5-Fluorouracil-loaded BSA nanoparticles: formulation optimization and in vitro release study. Aaps Pharmscitech. 2008; 9: 1092-1096.

18. Chowdhury EH. Nanotherapeutics: from laboratory to clinic: CRC press; 2016.

19. Kim BS, Oh JM, Kim KS, Seo KS, Cho JS, Khang G, et al. BSA-FITC-loaded microcapsules for in vivo delivery. Biomaterials. 2009; 30: 902-909.

20. Zhang C, Fu Y-Y, Zhang X, Yu C, Zhao Y, Sun S-K. BSA-directed synthesis of CuS nanoparticles as a biocompatible photothermal agent for tumor ablation in vivo. Dalton T. 2015; 44: 1311213118. 
OBM Genetics 2019; 3(3), doi:10.21926/obm.genet.1903090

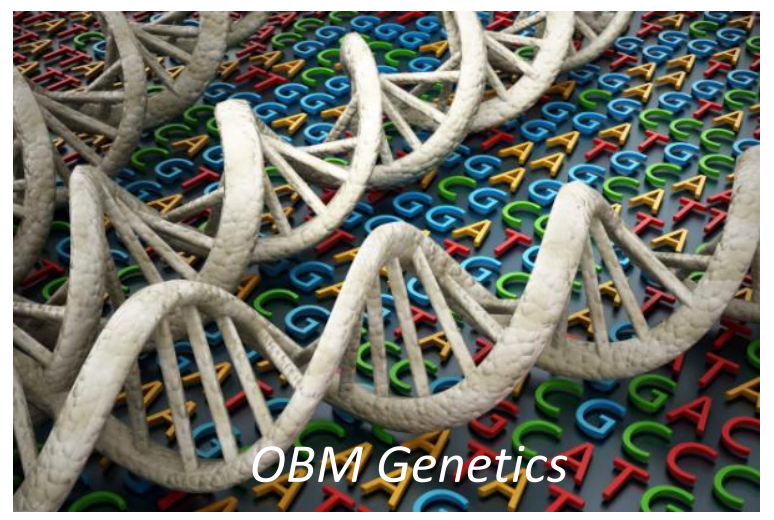

Enjoy OBM Genetics by:

1. Submitting a manuscript

2. Joining in volunteer reviewer bank

3. Joining Editorial Board

4. Guest editing a special issue

For more details, please visit:

http://www.lidsen.com/journals/genetics 